Research Article

\title{
Ultrasonography of the Vagus Nerve in the Diagnosis of Parkinson's Disease
}

\author{
Ovidijus Laucius $\mathbb{D D}^{1}{ }^{1}$ Renata Balnytè, ${ }^{1}$ Kęstutis Petrikonis, ${ }^{1}$ Vaidas Matijošaitis, ${ }^{1}$ \\ Neringa Jucevičiūtè $\mathbb{D}^{2}{ }^{2}$ Tadas Vanagas $\mathbb{D}^{2}{ }^{2}$ and Vytautas Danielius ${ }^{2}$ \\ ${ }^{1}$ Department of Neurology, Lithuanian University of Health Sciences, Eiveniu str. 2, LT-50009 Kaunas, Lithuania \\ ${ }^{2}$ Faculty of Medicine, Medical Academy, Lithuanian University of Health Sciences, Mickevičiaus str. 9, \\ LT 44307 Kaunas, Lithuania
}

Correspondence should be addressed to Ovidijus Laucius; ovidijus.laucius@gmail.com

Received 19 November 2019; Revised 18 February 2020; Accepted 9 March 2020; Published 31 March 2020

Academic Editor: Seyed-Mohammad Fereshtehnejad

Copyright (c) 2020 Ovidijus Laucius et al. This is an open access article distributed under the Creative Commons Attribution License, which permits unrestricted use, distribution, and reproduction in any medium, provided the original work is properly cited.

\begin{abstract}
Background. It is currently impossible to diagnose Parkinson's disease (PD) in the premotor phase even though at the time of motor symptom onset the number of already degenerated dopaminergic substantia nigra neurons is considerable. Degeneration of the dorsal nucleus of the vagus nerve $(\mathrm{VN})$ has been reported early in the disease course, and it could lead to impaired function of the VN, resulting in certain nonmotor symptoms of PD. Therefore, we raised a hypothesis that the loss of VN neurons could result in a smaller diameter of the VN among PD patients. Methods. $20 \mathrm{PD}$ patients and 20 age- and gender-matched individuals without any neurodegenerative disease were enrolled in a pilot study. The diameters of the right and left VNs were measured using ultrasonography, their average was calculated, and the narrower VN diameter was noted separately. Results. No difference was found between the PD and control groups neither in the average VN diameter (mean 1.17; 95\% confidence interval (CI) $1.10-1.24$ vs. $1.13 ; 1.07-1.18, \mathrm{~mm} ; p=0.353$ ) nor in the narrower VN diameter (mean $1.11 ; 95 \%$ confidence interval (CI) $1.02-1.20$ vs. 1.07; $1.02-1.13, \mathrm{~mm} ; p=0.421)$. The narrower $\mathrm{VN}$ diameter and the average $\mathrm{VN}$ diameter were not able to distinguish between $\mathrm{PD}$ patients and controls (area under curve $(\mathrm{AUC})=0.588,95 \% \mathrm{CI}=0.408-0.767$, and $p=0.344$; and $\mathrm{AUC}=0.578,95 \%$ $\mathrm{CI}=0.396-0.759$, and $p=0.402$ ). Conclusions. To conclude, no differences were found in VN diameter between the PD and control groups. Therefore, our data do not support the hypothesis that PD could be associated with a smaller diameter of the VN.
\end{abstract}

\section{Introduction}

$\mathrm{PD}$ is characterized by progressive neuronal loss in the substantia nigra (SN) pars compacta and widespread aggregation of the $\alpha$-synuclein protein that accumulates in Lewy bodies (LB) and Lewy neurites [1]. LB are detected in the SN, the locus coeruleus, the basal nucleus of Meynert, the cerebral cortex [2], and in many other parts of the brain of PD patients, including all cranial nerve nuclei, especially the dorsal motor vagal nucleus and the solitary nuclei [3-5]. Even though a possible neuroprotective role of LB has been suggested in some studies [2], LB have traditionally been considered toxic because the most significant neuronal loss is found in the predilection sites for LB, particularly in the
SN and locus coeruleus [2]. The loss of SN dopaminergic neurons results in dopamine depletion that leads to the typical motor symptoms of PD. Even though at the time of their onset up to $66 \%$ of SN dopaminergic neurons are already lost [4], it is currently impossible to diagnose PD in the premotor phase. Many potential disease-modifying agents are under research [5], and in case their efficacy is proven for the treatment of $\mathrm{PD}$, treatment should begin when neuronal loss is minimal; therefore, the ultimate goal would be to diagnose PD in the premotor phase; however, due to the unspecificity of the premotor symptoms, further testing is required. Developments in magnetic resonance imaging, positron emission tomography, and single-photon emission tomography raise the possibility of noninvasive 


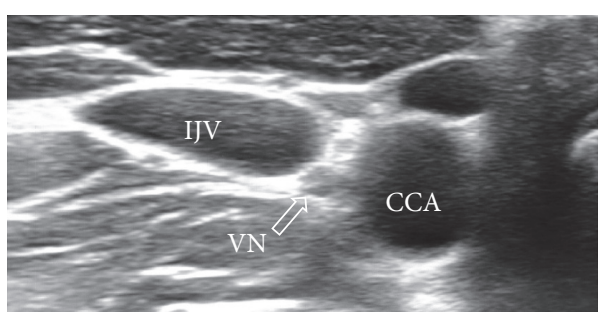

(a)

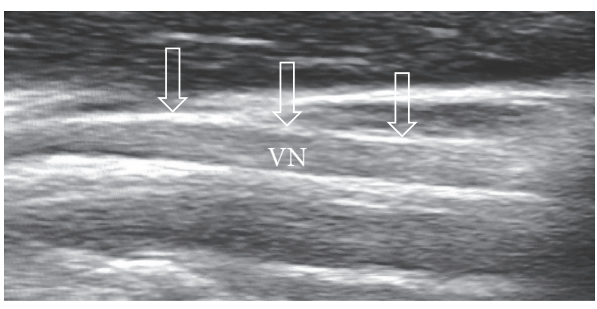

(b)

FIGURE 1: Ultrasonography scan of the vagus nerves in a healthy control subject. (a) Vagus nerve marked with an arrow in an axial image of the neurovascular bundle. (b) Vagus nerve marked with arrows in a sagittal projection. IJV, internal jugular vein; CCA, common carotid artery.

diagnostic testing for premotor PD [6]. Nevertheless, other potential premotor PD screening tests that are widely available, easy to use, and less expensive than these imaging methods should be investigated. One of these hypothetical methods is ultrasonography of the vagus nerve (USVN).

The accumulation of alpha-synuclein aggregates in some $\mathrm{VN}$ nuclei can be observed even at the earliest stages of PD, and it could result in impaired function of the VN, leading to the development of certain nonmotor symptoms of PD [7-9]. The loss of neurons in vagal nuclei could result in thinning of the $\mathrm{VN}$, detectable by ultrasound. A reduction of the cross-sectional area (CSA) of the $\mathrm{VN}$ in patients with $\mathrm{PD}$ has been recently shown in two studies $[10,11]$. Tsukita et al. reported significantly smaller CSA of the VN on both sides in PD patients $(n=21)$ compared with control subjects $(n=21)$ (right 1.58 vs. 2.35 and left 1.46 vs. $\left.1.91, \mathrm{~mm}^{2}\right)$ [10]. Walter et al. measured the VN CSA of 20 PD patients and 20 control subjects and reported similar findings (right 0.64 vs. 1.32 and left 0.69 vs. $1.12, \mathrm{~mm}^{2}$ ) [11]. However, in another study by Fedtke et al., no difference in the CSA between the groups was reported (PD group $(n=32)$ : left 2.6 , right 2.9 vs. healthy control subjects $(n=15)$ : left 2.4 , right $\left.2.7, \mathrm{~mm}^{2}\right)$ [12]. Despite the similar sample size of these three studies, the heterogeneity of the data is significant. Because the link between $\mathrm{PD}$ and the parameters of the $\mathrm{VN}$ remains uncertain, we aimed to compare the diameter of the VN between PD patients and healthy control subjects.

\section{Materials and Methods}

2.1. Study Design. Following approval by the Lithuanian University of Health Sciences Bioethics Committee, this study was conducted in the Hospital of Lithuanian University of Health Sciences Kaunas Clinics. All 40 patients gave informed consent to participate in the study. 20 PD patients and 20 age- and gender-matched individuals without any neurodegenerative disease were included. The diameters of the right and left VNs were measured using ultrasonography, their average was calculated, and the narrower VN diameter was noted separately. PD patients were also evaluated using the second and third parts of the Movement Disorder Society-Unified Parkinson's Disease Rating Scale (MDS-UPDRS). The stage of PD was evaluated using the modified Hoehn and Yahr scale.
TABLE 1: Clinical characteristics of PD patients.

\begin{tabular}{lcc}
\hline Clinical characteristics & PD patients $(n=20)$ \\
\hline Duration of PD, years & $4.0(1.0-6.25)$ \\
\hline MDS-UPDRS II, points & $8.5(7.0-18.0)$ \\
\hline MDS-UPDRS III, points & $25.0(21.3-32.8)$ \\
\hline & 1 & $1(5 \%)$ \\
& 1.5 & $4(20 \%)$ \\
Hoehn and Yahr stages of PD & 2.5 & $5(25 \%)$ \\
& 3 & $4(20 \%)$ \\
& 4 & $4(20 \%)$ \\
& 5 & $1(5 \%)$ \\
\hline
\end{tabular}

Values are given as median (interquartile range) or number (percentage); MDS-UPDRS, Movement Disorder Society-Unified Parkinson's Disease Rating Scale; PD, Parkinson's disease.

\subsection{Ultrasonography of the Vagus Nerve (USVN). The USVN} imaging was performed using a "Voluson 730 Expert" ultrasound device with a linear 6-12 $\mathrm{MHz}$ transducer probe, in a depth of $\sim 3.5 \mathrm{~cm}$. The imaging was performed with the patients resting on their back, with their head bent backward. The probe was placed in the transverse plane several centimetres above the medial ridge of the sternocleidomastoid muscle. The VN can be seen in ultrasound B-mode (Figure 1) near the bifurcation of the carotid artery, dorsal to the internal and common carotid arteries, surrounded by a sheet of connective tissue as a structure that is hypoechogenic in the centre and more hyperechogenic in the periphery $[13,14]$. VN diameter was measured in the sagittal plane in millimetres proximal to the carotid bulbus, at the level of the distal end of the common carotid artery.

\subsection{Statistical Analysis of Data. Statistical analyses were} performed using SPSS software package (version 25.0; IBM). Descriptive statistics were calculated. Data normality was assessed using histograms and the Shapiro-Wilk test. Nonparametric tests were used for non-normally distributed variables. The Student's $t$ and Mann-Whitney $U$ tests were used to evaluate the differences between the groups. The correlation between continuous variables was analysed using Spearman's rank correlation test. Receiver operating characteristic (ROC) curves were used to evaluate the diagnostic performance of the tests. All $p$ values are two-sided; The $p$ values less than 0.05 were regarded as statistically significant. 
TABLE 2: Comparison of age and VN diameter between the PD and control groups.

\begin{tabular}{|c|c|c|c|c|}
\hline & & PD group $(n=20)$ & Control group $(n=20)$ & $p$ value \\
\hline \multirow{3}{*}{ Age, years } & Median & 65.5 & 66.5 & \multirow{3}{*}{0.620} \\
\hline & IQR & $58.3-76.0$ & $60.5-76.3$ & \\
\hline & $95 \% \mathrm{CI}$ & $62.71-71.29$ & $63.99-71.51$ & \\
\hline \multirow{2}{*}{ Average $\mathrm{VN}$ diameter, $\mathrm{mm}$} & Mean & 1.17 & 1.13 & \multirow{2}{*}{0.353} \\
\hline & $95 \% \mathrm{CI}$ & $1.10-1.24$ & $1.07-1.18$ & \\
\hline \multirow{2}{*}{ Narrower VN diameter, $\mathrm{mm}$} & Mean & 1.11 & 1.07 & \multirow{2}{*}{0.421} \\
\hline & $95 \% \mathrm{CI}$ & $1.02-1.20$ & $1.02-1.13$ & \\
\hline
\end{tabular}

$\mathrm{CI}$, confidence interval; IQR, interquartile range; $\mathrm{PD}$, Parkinson's disease; SD, standard deviation; VN, vagus nerve.

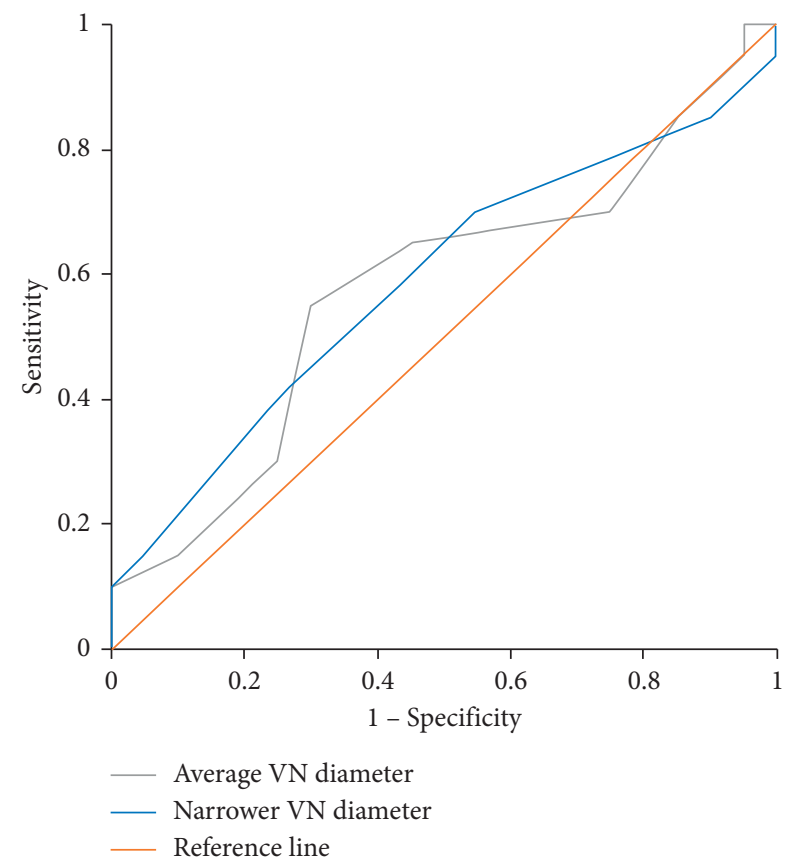

FIGURE 2: Discriminative abilities of the narrower and average vagus nerve diameters for Parkinson's disease. VN, vagus nerve.

\section{Results}

The study sample included 20 PD patients and 20 healthy controls. There were 10 females and 10 males in each group. The clinical characteristics of PD patients are presented in Table 1 . The majority of PD patients were mildly or moderately affected.

The age of PD patients and the control subjects did not differ (Table 2) and ranged from 55 to 81 years and from 54 to 80 years, respectively. No differences were found between the PD and control groups neither in the average diameter of the VN nor in the diameter of the narrower VN (Table 2).

In the control group, age did not correlate neither with the average diameter of the $\mathrm{VN}(r=-0.191$ and $p=0.420)$ nor with the diameter of the narrower VN $(r=-0.047$ and $p=0.843$ ).

The average $\mathrm{VN}$ diameter and the narrower VN diameter showed no statistically significant correlation with age, duration of disease, MDS-UPDRS II, or MDS-UPDRS III in the PD group (data not shown). Both the diameter of the VN on the narrower side and the average diameter on both sides were not able to distinguish between $\mathrm{PD}$ patients and healthy subjects (area under curve $(\mathrm{AUC})=0.588, \quad 95 \%$ $\mathrm{CI}=0.408-0.767$, and $p=0.344 ;$ and $\mathrm{AUC}=0.578,95 \%$ $\mathrm{CI}=0.396-0.759$, and $p=0.402$, respectively) (Figure 2 ).

\section{Discussion}

In this study, we observed no statistically significant difference in the diameter of the VN between PD patients and healthy controls even though VN diameter tended to be slightly greater in the PD group. Our results are in accordance with the ones reported by Fedtke et al. who also observed no significant differences in the VN CSA between controls and the PD group [12]. Our results are contrary to the ones reported in two other studies $[10,11]$. A few reasons could be explanatory of the varying results. First, the level chosen for the measurement of the $\mathrm{VN}$ was not identical. We measured $\mathrm{VN}$ diameter at the level of the distal end of the common carotid artery, and the same approach was chosen by Tsukita et al. [10]. Walter et al. measured VN CSA at the level of the thyroid cartilage [11], and Fedtke et al. did not indicate the exact level chosen for measuring the VN [12]. However, only this difference in methodology could not explain the fact that some studies found significant atrophy of the VN in PD patients, whereas this was not observed in others. Another reason that could explain the varying results is the difference in clinical characteristics of the PD group. However, VN CSA values reported by Walter et al. were a few times lower than the ones shown by Fedtke et al. despite similar age and MDS-UPDRS III score values $(30.7 \pm 12.4$ and $33 \pm 12)[11,12]$. The majority of patients were at stage II-III in our study as well as in the studies by Tsukita et al. and Fedtke et al. $[10,12]$. Because it seems that the clinical characteristics of patients did not differ so substantially that they could have resulted in such different measurements, the explanation of the heterogeneity of results remains unclear.

Studies exploring the association between VN CSA and age have also reported different results: a decrease in CSA among older subjects was reported in one study [15], whereas another one showed no association [16]. The results of our study were in accordance with the latter ones.

Even though the results of this study are important as now there are two studies that support the association between vagal atrophy and PD and two other studies with opposite results, the limitations of our study should be emphasised. First, only the diameter of the VN rather than both the diameter and the CSA was measured. Second, only 
one examiner evaluated the diameter of the VN. In addition, this was a single-centre study with a small sample size.

Because of heterogeneous results of all studies examining the association between the $\mathrm{VN}$ and $\mathrm{PD}$, further studies should be performed. We suggest performing a larger scale study that would include approximately equal percentages of $\mathrm{PD}$ patients in all stages of the disease. In order to ensure the reliability of vagal measurements, all subjects should undergo two ultrasonography evaluations by different physicians and both the diameter and the CSA of the VN should be recorded on both sides at different measurement levels.

\section{Conclusions}

In conclusion, it remains unclear whether PD is associated with structural changes of the $\mathrm{VN}$; therefore, although our results do not indicate the potential value of $\mathrm{VN}$ ultrasonography in the diagnosis of PD, future studies are warranted.

\section{Data Availability}

The underlying data related to this study are available from the corresponding author upon request.

\section{Conflicts of Interest}

The authors declare that there are no conflicts of interest regarding the publication of this article.

\section{Acknowledgments}

The publication of this article was funded by the Lithuanian University of Health Sciences.

\section{References}

[1] L. Xu and J. Pu, "Alpha-synuclein in Parkinson's disease: from pathogenetic dysfunction to potential clinical application," Parkinson's Disease, vol. 2016, pp. 1-10, 2016.

[2] K. Wakabayashi, K. Tanji, F. Mori, and H. Takahashi, "The Lewy body in Parkinson's disease: molecules implicated in the formation and degradation of $\alpha$-synuclein aggregates," $\mathrm{Neu}$ ropathology, vol. 27, no. 5, pp. 494-506, 2007.

[3] K. Seidel, J. Mahlke, S. Siswanto et al., "The brainstem pathologies of Parkinson's disease and dementia with Lewy bodies," Brain Pathology, vol. 25, no. 2, pp. 121-135, 2015.

[4] B. Pakkenberg, A. Moller, H. J. Gundersen, A. Mouritzen Dam, and H. Pakkenberg, "The absolute number of nerve cells in substantia nigra in normal subjects and in patients with Parkinson's disease estimated with an unbiased stereological method," Journal of Neurology, Neurosurgery \& Psychiatry, vol. 54, no. 1, pp. 30-33, 1991.

[5] A. E. Lang and A. J. Espay, "Disease modification in Parkinson's disease: current approaches, challenges, and future considerations," Movement Disorders, vol. 33, no. 5, pp. 660-677, 2018.

[6] T. R. Barber, J. C. Klein, C. E. Mackay, and M. T. M. Hu, "Neuroimaging in pre-motor Parkinson's disease," NeuroImage: Clinical, vol. 15, pp. 215-227, 2017.

[7] E. Lasser-Katz, A. Simchovitz, W.-H. Chiu et al., "Mutant $\alpha$-synuclein overexpression induces stressless pacemaking in vagal motoneurons at risk in Parkinson's disease," The Journal of Neuroscience, vol. 37, no. 1, pp. 47-57, 2017.

[8] C. H. Hawkes, K. Del Tredici, and H. Braak, "Parkinson's disease: a dual-hit hypothesis," Neuropathology and Applied Neurobiology, vol. 33, no. 6, pp. 599-614, 2007.

[9] H. Braak, K. Del Tredici, H. Bratzke, J. Hamm-Clement, D. Sandmann-Keil, and U. Rüb, "Staging of the intracerebral inclusion body pathology associated with idiopathic Parkinson's disease (preclinical and clinical stages)," Journal of Neurology, vol. 249, no. 3, pp. 1-5, 2002.

[10] K. Tsukita, T. Taguchi, H. Sakamaki-Tsukita, K. Tanaka, and T. Suenaga, "The vagus nerve becomes smaller in patients with Parkinson's disease: a preliminary cross-sectional study using ultrasonography," Parkinsonism \& Related Disorders, vol. 55, pp. 148-149, 2018.

[11] U. Walter, P. Tsiberidou, M. Kersten, A. Storch, and M. Löhle, "Atrophy of the vagus nerve in Parkinson's disease revealed by high-resolution ultrasonography," Frontiers in Neurology, vol. 9 , p. 805, 2018.

[12] N. Fedtke, O. W. Witte, and T. Prell, "Ultrasonography of the vagus nerve in Parkinson's disease," Frontiers in Neurology, vol. 9 , p. 525, 2018.

[13] F. Giovagnorio and C. Martinoli, "Sonography of the cervical vagus nerve," American Journal of Roentgenology, vol. 176, no. 3, pp. 745-749, 2001.

[14] N. Hammer, J. Glätzner, C. Feja et al., "Human vagus nerve branching in the cervical region," PLoS One, vol. 10, no. 2, Article ID e0118006, 2015.

[15] J. O. Pelz, E. Belau, P. Henn, N. Hammer, J. Classen, and D. Weise, "Sonographic evaluation of the vagus nerves: protocol, reference values, and side-to-side differences," Muscle \& Nerve, vol. 57, no. 5, pp. 766-771, 2018.

[16] M. S. Cartwright, L. V. Passmore, J.-S. Yoon, M. E. Brown, J. B. Caress, and F. O. Walker, "Cross-sectional area reference values for nerve ultrasonography," Muscle \& Nerve, vol. 37, no. 5 , pp. $566-571,2008$. 